\title{
A List of Plants collected in Soo-chow, China, by Prof. J. Matsumura and K. Ono.
}

by

\section{S. Matsuda.}

Mr. K. Ono, now a professor in the University of Pekin, collected plants in Soo-chow and its vicinity, while he was staying there. Afterwards, in 1908, he accompanied Prof. J. Matsumura in his short trip to that region, and a collection of plants was made by them. The plants thus collected were freely put in my hand, and the present list is the result of the examination of them. A few names of the plants in the list are new to the Flora of China, so far as I know; and also I was obliged to propose a new name for a species of Mosla which I have been unable to identify with any of the species described. These names are Swertia tosaensis Makino, Veronica polita Fries, Hygrophila lancea Miq., Mosla soochouensis n. s., Luzula campestris DC., var. intermedia Koidz. and Panicum Matsumurae Hack.

Here I express my sincere thanks to Prof. J. Matsumura and $\mathrm{K}$. Ono through whose kindness the materials for $\mathrm{my}$ study were obtained. Again thanks to Prof. J. Matsumura and others who helped me while I was examining these materials.

S. Matsuda.

In Bot. Inst. Sci. Coll. Imp. Univ. Tokyo.

Jan. 1912. 


\section{Dicotyledones.}

\section{A. Polypetalæ.}

Clematis grata WaLL.; Hook. f. Fl. Brit. Ind. I. 3 ; Max. in Mél. Biol. IX. 592; Forb. et Hemsl. in Journ. Linn. Soc. XXIII. 3 ; Diels in Engl. Bot. Jahrb. XXIX. $333 ;=C l$. vitalba L. II. subsp. \&. grata Wall., Kuntze, Monogr. 100. 天本山

The present specimen seems to be a variety of the species.

C. uncinata Champ; Benth. Fl. Hongk. 16 ; Max. Mél. Biol. IX. 597 ; Bot. Mag. Tokyo XX. $102 ;=$ C. recta L. III. subsp. $\xi$. chinensis Retz. var. uncinata Champ., Kuntze, Monogr. 115. 天本山

Delphinium anthriscifolium Hance; Bot. Mag. Tokyo 1. c. 上方山

Ranunculus acris L.; Tokyo Bot. Mag. 1. c. 103. 上方山

Akebia quinata Dene.; Bot. Mag. Tokyo. 1. c. 104; 上打山

Capsella Bursa-pastoris Moench.; Bot. Mag. Tokyo 1. e. 105. 上方山

Cardamine lyrata Bge.; Bot. Mag. Tokyo. 1.c. 上拈

C. sylvatica Link.; Max. in Mel. Biol. IX. 6 ; Forb. ct Hemsl. in Journ. Linn. Soc. XXIII. $43 ;=C$. hirsuta L. in Bot. Mag. Tokyo 1. c. 上方山

Moricandia sonchifolia Hook. f.; Bot. Mag. Tokyo. 1. c. =Orychophragmus sonchifolius Bge., Enum. Pl. Chin. Bor. 7 . 靈䉷山

It grows wild in abundance, and young leaves are said to be eaten.

Nasturtium globosum Turcz.; Hance in Journ. Linn. Soc. XIII. 76 ; Franch. P1. David. 31 ; Forb. et Hemsl. in Journ. Linn. Soc. XXIII. $39 ;=N$. austriacum in Bot. Mag. Tokyo 1. c., non Crantz. 上方山

Seed under magnification presents reticulation. In the present sp. stigma is much shorter than the globose fruit, but it is subequal in $N$. austriacum Crantz.

Nasturtium sikokianum Fr. et Sav.; Bot. Mag. Tokyo 1. c. 獅子山 
This is a species very closely allied to $N$. microspermum $D C$.

Viola Patrinii DC ; Benth. F1. Hongk. 20; Max. Mél. Biol. IX. 721. Forb. et Hemsl. in Journ. Linn. Soc. XXIII. 53; Diels in Engl. Bot. Jahrb. XXIX. 476. 上方山

Polygala sibirica L. ; Bot. Mag. Tokyo XX. 1806. 上方山

Arenaria leptoclados Guss.; Williams in Journ. Linn. Soc. XXXIII. 367. 蕉州

A. serpyllifola I. differs from the present species by having the sepals with 3 prominent nerves. Nerves are not distinct in A. leptoclados Guss.

Dianthus chinensis 1.; Rohrb. in Linnaea XXXVI. 670 ; Forb. et Hemsl. in Journ. Linn. Soc. XXIII. 63 ; var. $\beta$. silvaticus KocH, subvar. brachylepis Rohrb. 1.c.?

Dianthus superbus I. ; DC. Prodr. I. 365 ; Hance in Journ. Bot. 1883, 296 ; Frob. et Hemsl. in Journ. Linn. Soc. XXIII. 46. 蘇州

Silene aprica Turcz. var. $\alpha$. typica Rohrb. lusus 2 Rohrb.?; Bot. Mag. Tokyo. 1. c. 107. 獅子山

S. Fortunei VIs. ; Rohrb. in Linnaea XXXVI. 688 ; Forb. et Hemsl. in Journ. Linn. Soc. XXIII. 65 ; Matsum. et Hayata, Enum. P1. Formos. 35. 上方山, 天本山, 靈蟩山

Stellaria media L. ; Benth. Fl. Hongk. 21; Max. Mél. Biol. IX. 42 ; Forb. et Hemsl. in Journ. Linn. Soc. XXIII. 68. 上方山

Styles 3, seed flattened, orbiculor, echinate. It is said this species is naturalized in China from Europe.

Portulaca oleracea L.; DC. Prodr. III. 353; Benth. Fl. Hongk. 127 ; Forb. et Hemsl. in Journ. Linn. Soc. XXIII. 71. 蘇州

Grewia parviflora Bge.; Walp. Rep. 1. 360 ; Franch. Pl. David. 59 ; Forb. et Hemsl. in Journ. Linn. Soc. XXIII. 93 ; Diels in Engl. Bot. Jahrb. XXIX. 468. 天本山

Oxalis corniculata L.; Gray, Synop. Fl. N. America I. (1895 -1897), 365 ; Diels in Engl. Bot. Jahrb. XXIX. 420 ; Bot. Mag. Tokyo XX. 109. 上方山

O. stricta L. is an allied species, but the two are distinguished:

Fruiting pedicel refracted, longer than capsule, petiole stipulate. . . . . . . . . . . . . . . O. corniculata.

Fruiting pedicel not refracted, subequal to, or shorter than 
the capsule, petiole naked.

O. stricta.

Zanthoxylon alatum Roxв. ; Hook. f. Fl. Brit. Ind. I. 493; Forb. et Hemsl. in Journ. Linn. Soc. XXIII. $105 ;=Z$. planispinum Sieb. et Zucc., F1. Jap. Fam. Nat. 30 ; Franch. P1. David. 67. 蘇州

Ilex Oldhami Miq.; Forb. et Hems1. in Journ. Linn. Soc. XXIII. 117 ;=I. purpurea Hassk. o. Oldhami (Miq.) Loes. ; Diels in Engl. Bot. Jahrb. XXIX, 435. 天本山

Euonymus japonica Thunb.; Bot. Mag. Tokyo. XXI. 212. 蘇州

In the present specimen leaves are suborbicular, and some are emarginate.

Microrhamnus franguloides Max.; Forb. et Hemsl. in Journ. Linn. Soc. XXIII. $127 ;=$ Rhamnella franguloides Weberbauer; $=R h$. japonica Miq. in Ann. Mus. Bot. Lugd. Bat. III. 431 ;=Berchemia congesta S. Moore in Journ. Bot. 1875, 226. 天本山

Rhamnus parvifolius BGE.; Max. Rhamneæ orient. asia. 16 ; Franch. P1. David. 73 ; Forb. et Hemsl. in Journ. Linn. Soc. XXIII. 129 ; Diels in Engl. Bot. Jahrb. XXIX. 459. 上方山 Sageretia theezans Brongn.; Bot. Mag. Tokyo XX. 125. 蘇州

Zizyphus vulgaris Lam.; Bot. Mag. Tokyo 1. c. 天本山

Vitis heterophylla Thunb. Flora Jap. 103 ; Benth. F1. Hongk. 53 ; Forb. et Hemsl. in Journ. Linn. Soc. XXIII. 133 ; =Ampelopsis humulifolia Bge;=Cissus humulifolia Regel, Fl. ussur. t. 3. fig. 1.2, non Bunge; =Am. heterophylla Sieb. et Zucc.; Planch. in DC. Monogr. Phanerog. V. 455 ; Diels in Engl. Bot. Jahrb. XXIX. 465. 蕉州

This species is variable not only in the form of leaves, but also in the degree of pubescence. The present specimen is very pubescent in peduncles, petioles and other parts.

V. inconstans Miq.; Forb, et Hemsl. in Journ. Linn. Soc. XXIII. $133 ;=$ Parthenocissus tricuspida (S. et Z.) Planch.; Diels in Engler. Bot. Jahrb. XXIX. 464. 锚州

Acer palmatum Thunb.; Forb. et Hemsl. in Journ. Linn. Soc. XXIII. 141 ; Diels in Engl. Bot. Jahrb. XXIX. 448. 上方山 
A. Paxii Fr, var, ningponense (Hance) Pax in Pflanzenreich Heft 8, p. 10. 天不山

A. tataricum L.; DC. Prodr. I. 593 ; Forb. et Hemsl. in Journ. Linn. Soc. XXIII. 142; Diels in Engl. Bot. Jahrb. XXIX. 148;

var. Ginnala Max. F1. Amur. 67 ; Mél. Biol. X. 604; Fr. Pl. David. 76. 上方山

Rhus sylvestris Sieb. et Zucc., Fl. Jap. Fam. Nat. 32 ; Engl. in DC. Monogr. Phanerog. IV. 399 ; Forb. et Hemsl. in Journ. Linn. Soc. XXIII. 147 ; Diels in Engl. Bot. Jahrb. XXIX. 433; $=R h$. succedanea in Bot. Mag. Tokyo XX. 126, non Linn. 天本山

Crotalaria sessiliflora L., Bot. Mag. Tokyo XXI. 212. 葆州 Dulbergia hupeana Hance in Journ. Bot. (1882) 5 ; Forb. et Hemsl. in Journ. Linn. Soc. XXIII. 198. 天体山

Desmodium parvifolium DC. Prodr. II. 334; Baker in Hook. f. Fl. Brit. Ind. II. 174 ; Benth. Fl. Hongk. 84. ; Diels in Engl. Bot. Jahrb. XXIX. 414; =D. microphyllum DC. Prodr.

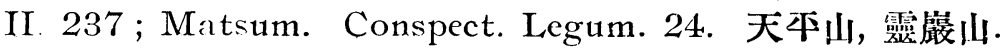

D. podocarpum DC. Prodr. II. 336 ; Baker in Hook. f. Fl. Brit. Ind. II. 165 ; Forb. et Hemsl. in Journ. Linn. Soc. XXIII. 174; ; Diels in Engl. Bot. Jahrb. XXIX. 414. 蘇州

Glycine Soja Sieb. et Zace; Bot. Mag. Tokyo XXI. 212. 蘇州

Indigofera venulosa Champ.; Walp. Ann. IV. 487 ; Benth. Fl. Hongk. 77 ; Forb. et Hemsl. in Journ. Linn. Soc. XXIII. $158 ;=I$. decora in Bot. Mag. Tokyo XX. 127, non Lindl. 獅子 山, 上方山, 靈蟩山

The two species are distinguished, thus:

Leaves ovate oblong, conspicuously glaucous on the back. I. decora Lindl.

Leaves ovate or suborbicular, not glaucous on the back......

I. venulosa Champ.

Lespedeza bicolor Turcz; Forb, et Hemsl. in Journ. Linn. Soc. XXIII. 179 ; Diels in Engl. Bot. Jahrb. XXIX. 415. 葆州

L. Buergeri Miq., Forb. et Hemsl. 1. c.; Diels 1. c. 天本山

L. juncea Pers. var. sericea Miq; Max. in Acta Horti 
Petrop. II. 368 (sp. propria); Bot. Mag. Tokyo XX. 127. 上方山, 天本川

L. striata Hook. et Arn.; Bot. Mag. Tokyo XXI. 283.

L. villosa Pers ; Bot. Mag. Tokyo XXI. 212. 蘇州

Medicago denticulata WILLD.; Forb. et Hemsl. in Journ. Linn. Soc. XXIII. 153; Diels in Engl. Bot. Jahrb. XXIX. 411; Matsum. in Bot. Mag. Tokyo XVI. 42. 天本山, 上方山

M. lupulina L. ; Bot. Mag. Tokyo XX 127 . 上方山

M. minina Lamk.; Led. F1. Ross. I. 520 ; Diels in Engl. Bot. Jahrb. XXIX. 411 ; Matsum. 1. c. 上方山, 霝簏山

Milletia reticulata Benth., Hance in journ. Linn. Soc. XIII. 161. Forb. et Hemsl. in Journ. Linn. Soc. XXIII. 159 ; Ito et Matsum. Tent. Fl. Lutch. 136 (or 403); Matsum. et Hayata Enum. P1. Formos. 105. 天不山

Phaseolus sp. 蘇州

Rhynchosia volubilis Lour; Bot. Mag. Tokyo XX. 226. 蘇州

Vicia hirsuta Koch.; Bot. Mag. Tokyo. XX. 127. 上方山

V. sativa L.; Bot. Mag. Tokyo. 1. c. 上方山

Agrimonia Eupatoria L.; Forb. et Hemsl. in Journ. Linn. Soc. XXIII. 246. 蘇州

Potentilla discolor Bge. Enum. P1. Chin. Bor. 25 ; Walp. Rep. II. 30 ; Forb. et Hemsl. in Journ. Linn. Soc. XXIII. 241 ; Diels in Engl. Bot. Jahrb. XXIX. 401. 上方山, 靈麗山

P. Fragarioides L. ; Bot. Mag. Tokyo XX. 128 . 上方山

P. supina L. ; Lehm. Revis. Potent. 193 ; Forb. ct Hemsl. in Journ. Linn. Soc. XXIII. 245; Diels in Engl. Bot. Jahrb. XXIX. 403 ; Luerssen in Bib. Bot. XVI. 389. 上方山

In my specimen mature carpels have distinct protuberance, and it seems to be of var. paradoxa Lucrs.

Rosa microcarpa Lindl.; Forb. et Hemsl. 1. c. 251; Diels 1. c. 405 . 蘇州

Rubus Thunbergii Sieb. et Zuce. Fl. Jap. Fam. Nat. no. 46 ; Max. in Mél. Biol. VIII. 389 ; Forb. et Hems1. 1. c. 238. 上方山

Sanguisorba officinalis L. ; Bot. Mag. Tokyo XX. 226. 天 本山, 鎮汇

Saxifraga sarmentosa L.; Forb. et Hemsl. 1. c. 268 ; Diels 
1. c. 364 . 蕉州

Cotyledon japonica Max.; Bot. Mag. Tokyo 1.c. 227. 蘇州 Sedum japonicum Sieb. ; Bot. Mag. Tokyo 1. c. 130. 上方山 Drosera peltata Sm. var. lunata Clarke; Bot. Mag. Tokyo 1. c. 靈祳山

Clarke distinguishes var. lunata from var. typica, thus:

Rosulate leaves persistent, sepals very fimbriate. . . . var. typica.

Rosulate leaves early deciduous, sepals erose or but slightly fimbriate. . . . . . . . . . . . . . . var. lunata.

Liquidamber formosana Hance; Bot. Mag. Tokyo 1.c. 天 平山

Ammania auriculata Willd.; DC. Prodr. III. 80 ; Koehne in Engl. Bot. Jahrb. I. 244 et IV. 389 ; Pritzel in Engl. Bot. Jahrb. XXIX. 483 ; Koehne in Engl. Pfl.Reich, Lythraceae 45. 蘇州

Hemsley in Journ. Linn. Soc. Bot. XXIII. 304 cites $A$. senegalensis Lam. with synonym A.auriculata Willd., and states: "We have followed Clarke in the synonymy of this species, as we have not critically examined the materials." However, after Koehne in Pfl. Reich 1. c. these two species are distinct, A. auriculata having the style longer than the ovary, while it is almost wanting in $A$. senegalensis. It is not certain true $A$. senegalensis is found in China or not.

Ludwigia prostrata RoxB.; Forb. et Hemsl. 1. c. 309 ; Diels 1. c. 484 . 上方山

Actimostemma racemosum Max.; Cogn. in DC. Monogr. III. 922 ; Forb. et Hems1. 1, c. 320. 蘇州

\section{B. Gamopetalae.}

Lonicera japonica Thunb. ; Bot. Mag. Tokyo. XX. 17. 蘇州

Imperfect specimen, determination unsatisfactory.

Galium aparine L.; Bot. Mag. Tokyo 1. c. 上方山

G. gracile BGE. Enum. Pl. Chin. Bor. 35 ; Forb. et Hemsl. in Journ. Linn. Soc. XXIII. 394; Diels in Engl. Bot. Jahrb. XXIX. 583 ; 
forma hispidum. 上方山

According to Bunge his plant is very smooth, but the present specimen is hispid.

Serissa Democritea Baill.; Bot. Mag. Tokyo XXI. $213 . \quad$ 天 本山

Patrinia scabiosaefolia Fisch.; DC. Prodr. IV. 624 ; Ledeb. Fl. Ross. II. 427 ; Fr. et Sav. Enum. Pl. Jap. I. 206 ; Forb. et Hems1. 1. c. 397 ; Diels 1. c. 597. 天本山

Artemisia capillaris Thunb.; Bot. Mag. Tokyo XX. 134. 蘇州

A. japonica Thunb.; DC. Prodr. VI. 100; Benth. Fl. Hongk. 186 ; Forb. et Hems1. 1. c. 443 ; Diels 1. c. 517 . 蘇州

Aster fastigiatum Fisch.; Bot. Mag. Tokyo XX. 227. 蘇州

A. indicus L. ;=Asteromaea indica Bl.; Bot. Mag. Tokyo 1. c. 134 . 上方山

A. trinervius Roxв.; Benth. Fl. Hongk. 174; Hook f. Fl. Brit. Ind. III. 252 ; Forb. et Hemsl. 1. c. 416 ; Diels 1. c. 610.

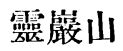

After Bentham this species is distinguished from an allied one $A$. baccharoides Steetz, thus :

Flower-heads hemispherical, on peduncles as long as or longer than themselves. . . . . . . . A. trinervius.

Flower-heads turbinato-campanulate, nearly sessile. . . .

A. baccharoides.

A. turbinatus S. Moore in Journ. Bot. 1878, 132 ; Forb.

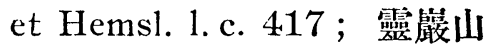

The peduncle with a number of bractlets gives to this plant an appearance of the species of Macroclinidium.

Bidens pilosa L. ; Bot. Mag. Tokyo XX. 134. 蘇州

B. tripartita L. ; DC. Prodr. V. 594; Hook. f. Fl. Brit. Ind. III. 309 ; Forb. et Hemsl. 1. c. 436 ; Diels 1. c. 616. 蘇州 蕉州

Carpesium abrotanoides L.; Bot. Mag. Tokyo XX. 227.

Chrysanthemum indicum L. ; Bot. Mag. Tokyo 1. c. 蘇州

Cnicus chinensis Benth.; Bot. Mag. Tokyo XXI. 214. 霝麗山

? C. Segetum (Bge.) Max. ; Bot. Mag. Tokyo XX. 134. 葆州 
Specimen imperfect, determination unsatisfactory.

Eclipta alba Hassk. ; Bot. Mag. Tokyo 1. c. 上方山

Eupatorium japonicum Thunв. ; DC. Prodr. v. 180 ; Fr. et Sav. Enum. Pl. Jap. I. 219 ; Forb. et Hemsl. 1. c. 403 ; Diels 1. c. 608 . 天本山

E. Lindleyanum DC. Prodr. v. 180. Benth. Fl. Hongk. 172 ; Forb. et Hems1. 1. c. 404; Diels 1. c. 608. 天本山

Gnaphalium hypoleucum DC.; Hook. f. F1. Brit. Ind. III. 288 ; Wight 1. c. t. 1114 ; Forb. et Hemsl. 1. c. 426 ; Diels 1. c. 613. 蘇州

G. japonicum Thuns. Fl. Jap. 311; Forb. et Hemsl. 1. c. 427. 獅子山, 上方山

G. multiceps Wall. ; Bot. Mag. Tokyo XX. 135. 獅子山

Inula britanica L.; DC. Prodr. V. 467 ; Fr. et Sav. Enum. Pl. Jap. II. 400 ; Forb. et Hems1. 1. c. 428 ; Diels 1. c. 614 . 上 方山

Diels cites the type and 2 var: var. japonica. Fr. et Sav. and var. linearifolia [linariaefolia?] Regel. The type after De Candolle has the back of leaves villose; and Franchet describes var. Japonica thus: "planta bi-pedalis glabrescens, folia ampla, lanceolata, intense viridia; rami virgata, elongati 1-3 cephali."-Banks Icon. Kaempf. tab. 30.

Senecio campestris DC. ; Bot. Mag. Tokyo XX. 135. 上方山 Solidago Virgaurea L. ; Bot. Mag. Tokyo XX. 227. 靈簏山 Xanthium strumarium L. ; Benth. Fl. Hongk. 181; Forb. et Hemsl. 1. c. 433.

Adenophora stricta Miq. ; Bot. Mag. Tokyo XX. 228. 蘇州

A. verticillata Fisch; Herder, Pl. Radd. IV. Heft 1. p. 28 ; Fr. et Sav. Enum. Pl. Jap. II. 422 ; Forb. et Hemsl. in Journ. Linn. Soc. XXVI. 14;

?var. triphylla Miq. (=var. subintegrifola Regel) in Zotei Somoku Zusetsu Vol. III. p. 11. 天本山

Lobelia radicans Thunb. ; Bot. Mag. Tokyo 1. c. 137. 蕉州

Platycodon Grandiflorus A. DC. ; Forb. et Hemsl. 1. c. 5 ; Diels in Engl. Bot. Jahrb. XXIX. 607. 天本山

Rhododendron sinense Sw. ; Bot. Mag. Tokyo 1. c. 上方山

Rh. Weyrichi Maxim. Rhod. As. Orient. 26, t. 2 fig. 1-6 ; 
Fr. et Sav. Enum. P1. Jap. I. 288 ; Forb. et Hemsl. 1. c. 32 ;= Rh. Farrerae Tate, $\alpha$. Weyrichi Max. 上方山

Stamens 10, upper throat maculated, tegument obscurely mucronate. It is distinguished from allied sp., thus :

Stamens 5. . . . . . . . . . . . Rh. dilatatum Miq.

Stamens 10, corolla not maculated. . Rh. rhombicum Miq.

Stamens 10, the upper throat of corolla maculated :

Leaves twin (always?) on the tip of the shoot, tegument mucronate. . . . . . . . . . . Rh. Weyrichi. Max.

Leaves three on the tip of the shoot, tegument rotund.

Rh. Farrerae Tate.

Vaccinum bracteatum Thunb. ; Bot. Mag. Tokyo 1. c. 天平山

Lysimachia candida Lindl.; Bot. Mag. Tokyo 1. c. 靈厥山, 上方山

L. Fortunei Max. ; Hance in Journ. Bot. VIII. (1870) 275 ; Somoku Zusetsu ed. 2. Vol. III. fol. 64; Forb. et Hems1. 1. c. 52 ; Pax et Knuth in Engl. Pfl.Reich Heft 22 (Primulaceae) 290. 蘇州

L. Klattiana Hance ; Bot. Mag. Tokyo 1. c. 228. 上方山 Ardisia japonica B1. ; Bot. Mag. Tokyo 1. c. 138. 蘇州

Styrax serrulatus RoxB.; DC. Prodr. VIII. 267; Forb. et Hems1. 1. c. 77 ; Diels 1. c. 530 ; Perkins in Engl. Pfl.Reich Heft 30 (Styracaceae) 36. 上方山

Symplocos crataegoides Buch.-Ham.; Bot. Mag. Tokyo 1.c. 138. 上小方

Fontanesia phillyreoides Labill. ; Bot. Mag. Tokyo 1. c. 139. 天平山

In this species we often find abnormal friuts produced by the unuusual growth of the ovary with style. Hance described this as "the curious rapidly growing abortive ovary of the sterile form."-Journ. Bot. (1879)136.

Trachelospermum jasminoides Lemaire; Bot. Mag. Tokyo 1.c. 上方山

Gentiana squarrosa LEDEB. Fl. Ross. III. 64; Forb. et Hemsl. 1. c. 135 ; Diels 1. c. 537. 上方山

Swertia (Ophelia) tosaensis Makino in Bot. Mag. Tokyo XVII. (1903)54; $=$ S. chinensis Franch. var. tosaensis Makino in 
Bot. Mag. Tokyo VI. (1892)53. 靈笪山

This species is new to the Chinese Flora. Its stigma is described as bifid; but this is not clearly noticed in the present specimen.

Bothriospermum Kusunezowii Bge. ?; DC. Prodr. X. 116 ; Max. in Mél. Biol. VIII. 560 ; Forb. et Hems1. 1. c. 151 ; Diels 1. c. $546 ;=$ Thyrocarpus Sampsoni in Bot. Mag. Tokyo 1.c. 140, non Hance. 上方山, 獅子小

B. tenellum Fisch, et Mey. ; Bot. Mag. Tokyo 1. c. 140 ;= B. aspergoides Sieb. et Zucc. Fl. Jap. Fam. Nat. II. 150. 上方山

Lithospermum arvense Linn.; Bot. Mag. Tokyo 1. c. 上方山

L. Zollingeri A. DC. Prodr. X. 587 ; Forb et Hemsl. 1. c. 155 ; Diels 1. c. 546 . 上方山

Trigonotis peduncularis Benth.; Bot. Mag. Tokyo 1. c. 上方山

Lycium chinense Mill.; Bot. Mag. Tokyo 1. c. 229. 蕉州

Physalis minima L.; DC. Prodr. XIII. 1. p. 445 ; Clarke in Hook. f. Fl. Brit. Ind. IV. 238 ; Forb. et Hemsl. 1.c. 174; Leveille in Bull. Soc. Bot. Fr. Tom. 55, 208. 蘇州

Solanum lyratum Thunb. ; Bot. Mag. Tokyo 1. c. 蘇州

Mazus stachydifolius Max. Mél. Biol. IX. 404; Forb. et Hems1. 1. c. 184 ;=M. villosus Hemsl. in Journ. Bot. (1876)209 ; =Vandellia stachydifolia Walp. Rep. III. 294; DC. Prodr. X. 417. 上方山

Vandellia angustifolia Bentr. ; DC. Prodr. X. 417 ; Hook. f. F1. Brit. Ind. IV. 282 ; Forb. et Hemsl. 1. c. 189; Diels 1. c. 567. 上方山

Veronica polita Fries.; Halàcsy, F1. Graecae II. 435 ; Thomé, Fl. Deutsch. IV. 173 t. $533 \mathrm{~B} ;=$ V. agrestis in Bot. Mag. Tokyo XX. 141, non Linn. 上方山

V. spuria L. ; Ledel. Fl. Ross. III. 231 ; Forb. et Hemsl. 1. c. 200 ; Diels 1. c. $567 ;=V$. paniculata L.; Benth. in DC. Prodr. X. 465. 天本山

Utricularia sp. 蘇州

Tecoma grandiflora Loiseleur ; DC. Prodr. IX. 223 ; Forb. et Hemsl. 1. c. 235. 蘇州

Trapella sinensis OLIV. in Hook. Ic. P1. t. 1595 ; Forb. et 
Hemsl. 1. c. 236 ; Diels 1. c. 578. 靈蟩山

Hygrophila lancea Mro. Prol. 55 ; Fr. et Sav. Enum. P1. Jap. I. 355 ; =Justicia lancea Thunb. 蘇州

$H$. salicifolia Ness. reported from China by several authors is closely allied to the present species, but it has the throat of the corolla barbate, which is not the case with $H$. lancea. If my identification is right, the species is new to the Chinese flora.

Justicia procumbens L. ; Bot. Mag. Tokyo XXI. 215. 蕉州

Clerodendron trichotomum Thunв.; DC. Prodr. XI. 668 ; Bot. Mag. t. 6561 ; Forb. et Hemsl. 1. c. 262 ; Diels 1. c. 550. 蘇州

Verbena officinalis L. ; Bot. Mag. Tokyo XX. 142 蘇州

Vitex Negundo L. ; Bot. Mag. Tokyo 1. c. 天平山

Ajuga genevensis L. ; DC. Prodr. XII. 596 ; Max. in Mél. Biol. XI. 815 ; Forb. et Hemsl. 1. c. 315 ; Diels 1. c. 550, et XXXIV. Beibl. Nr. 75, p. 62 . 上方山, 獅子川

Elsholzia cristata WILLD. ; Benth. in DC. Prodr. XII. 163 ; Hook. f. Fl. Brit. Ind. IV. 645 ; Bot. Mag. t. 2550 ; Forb. et Hems1. 1. c. 277 ; Diels 1. c. 560. 靈祳山

The present specimen has much smaller leaves than the Japanese one. It seems to be of forma saxatilis Kom., Fl. Mans. III. 390.

Lamium album L. ; Bot. Mag. Tokyo 1. c. 143. 蘇州

Leonurus sibiricus L. ; Bot. Mag. Tokyo 1. c. 天本山

Mentha arvensis L.; Benth. in DC. Prodr. XII. 171; et F1. Hongk. 276 ; Hook. f. Fl. Brit, Ind. IV. 648; Forb. et Hemsl. 1. c. 281 ; Diels 1. c. 559 ; Somoku-Zusetsu 2. ed. XI. fol. 28. 蘇州

Mosla grosseserrata Max. in Mél. Biol. IX. 432 ; Forb. et Hemsl. 1. c. 280. 天本山

M. punctata Max. 1. c. 436 ; Forb. et Hemsl. 1. c. 281 ; Diels 1. c. 560. 上方山

Specimen very imperfect, but I notice 3 teeth of the upper lip of the calyx are subequal; this is an important character of the present species.

M. soochouensis $n$. $s$.

Herb, annual?, 3-4 dm. high, pubescent, stem 4-angled, 
branching; leaves opposite, lanceolate, shortly petiolate (petiole $5 \mathrm{~mm}$. long), subacuminate, subcuneate at base, serrate, glabrous, densely punctated with glands and glaucous below, (lamina $2-3 \times .6-.8 \mathrm{~cm}$.) ; racemes terminal, elongated, loose flowered; bracts minute, not exceeding fruiting pedicel, suborbicular, cuspidate ; flowers about $6 \mathrm{~mm}$. in length ; calyx pubescent, slightly 2-labiate, or 5 lobed, lobes subequal, slightly gibbose in fruit; corolla about twice as long as calyx, 2-labiate, upper lip erect emarginate, lower one 3-lobed, the median lobe largest ; stamens 4 , posterior or upper 2 fertile, subexserted, with cells of anthers divergent, anterior or lower 2 sterile, included; ovary deeply 4-parted, style subexserted, 2-parted above, branches subequal; nucules brownish, globose, reticulated, densely covered with minute dotes, furnished with a protuberance at base.

Souchow. (蕉州)

M. lanceolata Max. seems to be an allied species, but it differs from the present one by having the lanceolate brats exceeding pedicel.

Nepeta Glechoma Benth.; Bot. Mag. Tokyo XX. 143. 靈㦑山

Salvia japonica Thunb. var integrifolia Fr. et Sav.?; Bot. Mag. Tokyo XXI. 215. 天本山

Salvia miltiorhiza Bge.; Benth. in DC. Prodr. XII. 177 ; Forb. et Hems1. 1. c. 280 ; Diels 1. c. 557.=S. pogonocalyx. Hance in Journ. Linn. Soc. XIII. 85. 蘇州

S. plebeia R. Br. ; Bot. Mag. Tokyo XX. 143. 蘇州

Scutellaria indica L.; Bot. Mag. Tokyo 1. c. 144. 上方山, 獅子山

S. rivularis Wall. ; Benth in DC. Prodr. XII. 426; Wight Ic. P1. Ind. Or. t. 1450 ; Hooker f. F1. Brit. Ind. IV. 670 ; Matsum. et Hayata, Enum. P1. Formos. 314 ; Bot. Mag. Tokyo 1. c. 蘇州

According to Wight the flower is white, but Hooker states it is blue. In my specimen it is blue too. Wight's figure represents the leaves as entire, but Bentham describes them as crenate. 


\section{Monochlamydeae}

Alternanthera sessilis R. Br.; Bot. Mag. Tokyo XX. 144. 蘇州

Amarantus paniculata L.; Moq. in DC. Prodr. XIII. 2. p. 257 ; Hook. f. Fl. Brit. Ind. IV. 718 ; Forb. et Hemsl. in Journ. Linn. Soc. XXVI. 320 ; Diels in Engl. Bot. Jahrb. XXIX. 316. 蕉州

Chenopodium album L. ; Bot. Mag. Tokyo 1. c. 145. 䔡州 Polygonum aviculare L.; Bot. Mag. Tokyo 1. c. 蘇州

P. flaccidum MeIsn. in DC. Prodr. XIV. pro parte; Hook. f. F1. Brit. Ind. V. 39 ; Forb. et Hemsl. 1. c. 339. 蘇州

P. japonicum Meism ; Bot. Mag. Tokyo 1. c. 231 蘇州

P. lapathifolium L.; Hook. f. Fl. Brit. Ind. V. 25. 蘇州 var. incanum Ledeb. F1. Ross. III. 521; Bot. Mag. Tokyo 1. c. 145 . 上方山

P. Orientale L. ; Bot. Mag. Tokyo 1. c. 蘇州

P. Posumbu Hamil. ? Forb. et Hemsl. 1. c. 346 ; Diels 1. c. 312 ; Nakai in Bot. Mag. Tokyo XXIII. 394 (in Japanese). 蘇州

Specimen not very good, determination unsatisfactory.

P. strigosum R. Br.; Meisn. in DC. Prodr. XIV. 134; Hook. f. Fl. Brit. Ind. V. 47 ; Forb. et Hems1. 1. c. 350. 蕉州

P. Thunbergii Sieb. et Zucc. Fl. Jap. II. 84; Meisn. in DC. Prodr. 1. c. 132 ; Fr. et Sav. Enum. Pl. Jap. II. 475 ; Forb. et Hemsl. 1. c. 351 ; Diels 1. c. 314 ;

var. Maackiana (Regel) Max. ; Fr. et Sav. 1. c. 蘇州

var. hastato-triloba Max. is almost indistinguishable from the present specimen when we follow the description; but Franchet and Savatier refer var. hastato-triloba to Somoku Zusetsu Vol. VII. fol. 47, and Honzo Zufu Vol. XL. fol. 14 recto, both of which represent a plant differing from the present one.

P. viscosum HaM.; Meisn. 1. c. 102 ; Benth. F1. Hongk. 287 ; Hook. f. Fl. Brit. Ind. V. 36 ;

var. minor Ноок. f. 1. c. ? 上方山

Herb, 4 or $5 \mathrm{dm}$. high, branched, pili patent, leaves ovate subacute, cuneate at base, then gradually attenuated and form- 
ing wings to tne petiole, sheath short, densely covered with pili, peduncles glandular, spike densely flowered, shorty cylindrical, perianth 5 , purplish, stamens 5 ?, nut trigonose, chestnut colored, style 3 .

Rumex acetosa L. ; Bot. Mag. Tokyo XX. 146. 蕉州

Lindera glauca Bl. ; Bot. Mag. Tokyo 1.c. 上方山, 天本山

Elaeagnus pungens Thunb; Bot. Mag. Tokyo 1.c. 147. 蘇州

This species differs from an allied one E. Bockii Diels by having longer peduncles (more than $5 \mathrm{~mm}$.). The peduncle is said to be $1-2.5 \mathrm{~mm}$. in E. Bockii.

Thesium chinense Turczu ; Bot. Mag. Tokyo 1. c. 148 . 上方 山

Euphorbia Esula L. Boiss. in DC. Prodr. XV. 2. p. 160 ; Sow. Eng. Bot. VIII. 107 ; Forb. et Hemsl. in Journ. Linn. Soc. XXVI. 412 ; Diels in Engl. Bot. Jahrb. XXIX. 431. 蕉州

Glochidion Fortnni Hance in Ann. Sc. Nat. 4 ser. XVIII. 298 ? 天本山

Mallotus Apelta Muell. Arg. in Linnaea XXXIV. 189, et in DC. Prodr. XV. 2. p. 963 ; Forb et Hems1 1. c. 439 ; Diels 1. c. $428 ;=M$. japonicus in Bot. Mag. Tokyo XX. 164, non Muell. Arg. ;=Rottlera chinensis Juss. ; Hook. et Arn. Bot. Beech. Voy. 212 ; Benth. Fl. Hongk. 306. 本天山

Phyllanthus simplex Retz.; DC. Prodr. XV. 2. p. 391 ; Hook. f. Fl. Brit. Ind. V. 295 ; Forb. et Hemsl. 1. c. 423 ; Diels 1. c. 427 ; Hayata in Journ. Sci. coll. Imp. Univ. Tokyo vol. XX. Art. 7. p. $10 ;=$ Ph. anceps Vahl.; Benth. F1. Hongk. 331. 蘇州

Sapium sebiferum Rorb.; Bot. Mag. Tokyo XX. 232. 蘇州 Aphananthe aspera Planch. ; Bot. Mag. Tokyo 1. c. 蘇州 Celtis sinensis Pers? ; Bot. Mag. Tokyo 1. c. 165 上方山 Ulmus parvifolia Jacq. ; Bot. Mag. Tokyo 1. c. 233. 蘇州

Platycarya strobilacea Sieb. et Zucc. ; Bot. Mag. Tokyo 1.c. 166. 天本山, 上方山

Pterocarya stenoptera DC. ; Bot. Mag. Tokyo 1. c. 蘇州 Quercus aliena B1. ; Bot. Mag. Tokyo 1. c. 166. 蘇州

Q. Fabri Hance in Journ. Linn. Soc. X. 202 ; et in Journ. 
Bot. 1875, 362 ; Forb. et Hemsl. 1. c. 512. 天本山

\section{Monocotyledones.}

Platanthera interrapta Maxim.; Bot. Mag. Tokyo XX. 168. 靈䉷山

Spiranthes australis Lindl. ; Bot. Mag. Tokyo 1. c. 天本山

Liriope spicata Lour. ; Bot. Mag. Tokyo XX. 235. 天本山

Iycoris radiata Herb.; Kunth. Enum. V. 546 ; Max. in Engl. Bot. Jahrb. VI. 78 ; Wright in Journ. Linn. Soc. 89. 蘇州

Allium nipponicum Fr. et Sav.; Bot. Mag. Tokyo 1. c. 169. 靈耑山

This species is not yet reported from China by European authors. Unfortunately my material is not ample and even good.

Hemerocallis sp. 天本山

Scilla chinensis BentH. Fl. Hongk. 373 ? ; Bak. in Journ. Linn. Soc. XIII. 233 ; Wright in Journ. Linn. Soc. XXXVI. 127 ; Diels in Engl. Bot. Jahrb. XXIX. 245 ; Komarov. in F1. Mans. I. 465 ;=Barnardia scilloides Lindl. ; Hook. Bot. Mag. t. 3788 ; Kunth. Enum. P1. IV. 337. 蘇州

The present species seems to be closely allied to or even conspecific with S. japonica; but the latter is not hitherto reported from China Proper, while the former is.

Aneilema Keisak Hassk. Commel. Ind. 32 ; Clarke in DC. Monogr. Phan. III. 207 ; Brown in Journ. Linn. Soc. XXXVI. $152 ;=A$. oliganthum Fr. et Sav. Enum. P1. Jap. II. 94. et 532. 蘇州

Commelina communis L. : Benth. F1. Hongk. 376 ; Clarke in DC. Monogr. Phan. III. 170 ; Brown in Journ. Linn. Soc. XXXVI. 155, Diels in Engl. Bot. Jahrb. XXIX. 237. 上方山

Juncus alatus Fr. et Sav.; Bot. Mag. Tokyo XX. 169. 上方山

J. bulbosus L.?; Kunth. Enum. P1. III. 351 ; Brown in Journ. Linn. Soc. XXXVI. 163 ; =J. compressus Jacq. ; Buchenau in Engl. Bot. Jahrb XII. (1890)185 ; Sow. Eng. Bot. X. 37, t. 1575. 蕉州

Determination unsatisfactory. 
J. effusus I. ?; Bot. Mag. Tokyo 1.c. 170. 蕉州

Luzula campestris DC.; Bot. Mag Tokyo 1. c.

var. intermedia Kordz. in Journ. Col. Sci. Imp. Univ. Tokyo XXVII. Art. 13 (Plantae Sachalinenses Nakaharanae), 32. 蘇州 This variety is new to the Chinese Flora.

Arisaema japonscum B1. ; Kunth. Enum. III. 16 ; Ingl. in DC. Monogr. Phan. II. 539 ; Diels in Engl. Bot. Jahrb. 1. c. 236; Brown 1. c. 178. 蘇州

The specimen lacks both $\mathrm{fl}$. and fr.; determination unsatis. factory.

Alisma Plantago L. var. angustifolium Kunth; Enum; Pl. III. 148 ; Wright in Journ. Linn. Soc. XXXVI. 189. 天否山

Sagittaria sagittifolia L.; Kunth. Enum. Pl. III. 156; Diels in Engl. Bot. Jahıb. 1. c. 220; Wright 1. c. 190. 蘇州

- Carex breviculmis R. Br. Bot. Mag. Tokyo XX. 171. 州蕉

C. (Rhomboidales Kük.) laticeps C. B. Clarke?; Diels 1. c. 232; Clarke in Journ. Linn. Soc. 1. c. 293 ; Kükenthal in Pfl.Reich, Heft 38 (Caricoid.) 633. 上方山

Rhizome thick, lignose, culms 3-4 dm. high, smooth, leafy at base ; leaves shorter than culms, 4-6 mm broad, coriaceous, pubescent; spikes 2 , rarely 3 , the terminal $\hat{\delta}$, cylindrical or subclavate, $2.5-3 \mathrm{~cm}$. long, the lateral $q$, cylindrical $3 \mathrm{~cm}$. long, $8 \mathrm{~mm}$. across, shortly pedunculate, bracts with long. vagina and short lamina; scales of the female spike ovate, acuminate, whitish, with distinct green midnerve, hispido-pubes cent; utricle ovoid, turgiclo trigonal, $6 \mathrm{~mm}$. long (incl, rostrum), olivaceus, hispido pubescent, multinerved, rostrum subcylindrical, nearly as long as the body of the utricle, mouth shortly bifurcate; achenium trigonose, constricted in the middle, crowned with the thickened remains of the basal portion of style; stigma 3 .

Kuikenthal describes the margin of rostrum as setose, but in my specimen it is simply hispidulous.

C. neurocarpa Max.; Bot. Mag. Tokyc 1. e. 172. 上方山

C. nubigena Don.; Bot. Mag. Tokyo 1. c. ;

var. albata (Boott) Kük. forma laxiuscula Kǘ. 1. c. 146.? 上方山 
Kükenthal states about this forma: "spicula [spica?] clongata, cylindrica, subinterrupta." The present specimen is certainly of var. albata and probably of forma laxiuscula.

C. tristachya Thunb. ; Boott, Carex IV. 131, t. 424; Diels in Engl. Bot. Jahrb. XXIX. 231; Clarke in Journ. Linn. Soc. XXXVI. 315 ; Kiik. 1. c. 471. 蘇州

In this specimen the scales of the female spike are not mucronate; and those of the male not cup-shaped. It is certainly not of var. pocilliformis (Boott) Kük., as in this variety male scales are cup-shaped.

Cyperus difformis L.; Benth. F1. Hongk. 385 ; Kunth. Enum. Pl. II. 38 ; Clarke in Hook. f. Fl. Brit Ind. VI. 599 ; et 1. c. 210 . 蘇州

C. Iria L.; Kunth. Enum. Pl. II. 38 ; Benth. Fl. Hongk. 386 ; Clarke in Hook. f. Fl. Brit. Ind. VI. 606 ; Diels in Eng!. Bot. Jahrb. XXIX. 227 ; Clarke in Journ Linn. Soc. XXXVI. 213. 蘇州

C. sp. 天本山

A specimen of a tall Cyperus consisting of the upper portion of the plant. ; flowers not fully developed. Quite indeterminable.

Fimbristylis diphylla VAHL.; Benth. Fl. Hongk. 392 ; C. B. Clarke in Hook. f. Fl. Brit. Ind. VI. 636 ; Diels in Engl. Bot* Jahrb. XXIX. 129 ; Clarke 1. c. 233. 蘇州

This species is variable; and some authors distinguish several varieties.

E. miliacea Vahl.; Benth. Fl. Hongk. 393; Clarke in Hook. f. F1. Brit. Ind. VI. 744 ; Diels 1 c. 229 ; Clarke in Journ. Linn. Soc. XXXVI. 239. 蘇州

Pycleus sanguinolentus NeEs.; C. B. Clarke in Hoỏk. f. Fl. Brit. Ind. VI. 599, et in Journ. Linn. Soc. 1. c. 206. 蘇州

Rhynchospora glauca VAHL. var. $\beta$. chinensis C. B. Clarke in Hook. f. Fl. Brit. Ind. VI. 672, et in Journ. Linn. Soc. 1. c. $259 ;=R h$. chinensis Boeck. in Linnaea XXXVII. 586 (non Nees et Meyer). 天本山

My specimen is not very good ; determination unsatisfactory. Spikelets $1 / 4$ in. long, beak equaling nut in length, hypogynous bristles reaching top of the beak. In the type of the species, 
spikelets have a little shorter, hypogynous scales not reaching the top of the beak, (specim. in our Herb. det. by Clarke seen).

Rh. Wallichiana Kunth. Enum. Pl. II. 290 ; C. B. Clarke in Hook. f. Fl. Brit. Ind. VI. 668; et in Journ. Linn. Soc. 1. c. 260. 天本山

Scleria hebecarpa NeEs ; Kunth 1. c. 357 ; Boeck. in Linnaea XXXVIII. 478 ; Clarke in Hook. f. Fl. Brit. Ind. VI. 689 ; et in Journ. Linn. Soc. 1. c. $264 ;=S$. japonica Stend. Cyper. 169. 天本山

Agrostis perennans Tuck.; Bot. Mag. Tokyo XX. 173. 上方山

Alopecurus aequalis SoboL.; Rendle in Journ. Linn. Soc. XXXVI. 384; $=A$. fulvus Smith; Bull. Herb. Boiss. VII. (1899) $648 ;=A$. geniculatus L. ; Pilger in Engl. Bot. Jahrb. XXIX (1.900)224. 蘇州

A. japonicus Steud. ; Bot. Mag. Tokyo 1.c. 上方山

Beckmannia erucaeformis Host.; Bot. Mag. Tolsyo 1.c. 上方山

Cymbopogor Nardus Rende subsp. marginatus var. Goeringii HACK. ; Rendle in Journ. Linn. Soc. 1. c. 376 ;=Andropogon Nardus I. subsp. marginatus var. Goeringii Hack. Monogr. Androp. 607. 靈祳山

Eremochloa ophiuroides Hack. Monogr. Androp. 261; Rendle 1. c. $363 ;=$ Ischaemum ophiouroides Munro; Benth. Fl. Hongk. 425. 蘇州

Ophiurus monostachyus Presl. is apparently similar to the present species.

Ischaemum ciliare RETz. ; Hook. f. Fl. Brit. Ind. VII. 134; Hack. in Bull. Herb. Boiss VII. (1899)723; Rendle 1. c. 365 蘇州

Panicum acroanthum Steun. Sypop. Gram. 87 ; Hack. in Engl. Bot. Jahrb. VI. (1884) 49, et in Bull. Herb. Boiss. VII. (1899)544, et sér. 2, III. (1903)302 ; Rendle 1. c. 327. 蘇州

P. indicum L.; Benth. Fl. Hongk. 413. Pilger in Engl. Bot. Jahrb. XXIX. (1900)223 ; Rendle 1. c. 330 . 蕉州

Rendle 1.c. distinguishes 2 varieties, namely: angustatum Hook. f. Fl. Brit. Ind. VII. 42, and contractum Miq. in Ann. 
Mus. Bot. Lugd.-Bat. II. 275, But according to Hooker, var. angustatum is mere "starved form" of the species; and var. contractum is not distinguished from the type by Miquel, and also. by Franchet and Savatier.

P. Matsumurae Hack. in Bull. Herb. Boiss. (1899)644; Matsum. Index. Pl. Jap. Phanerog. pars 1(1905), $70 ;=$ Setaria excurrens Miq. Prol. 163 (non Trin). 天本山

So far as I know this name has not been reported from China.

Setaria Forbesiana Hook. f. apparently resembles the present spcies.

Pasparum scrobiculatum I.; Benth. Fl. Hongk. 408; Pilger in Engl. Bot. Jahrb. XXIX. 223 ; Hook. f. Fl. Brit. Ind. VII. 10 ; Rendle 1. c. $320=P$. Thunbergii Kunth. (sec. Rendle).

Rottboellia compressa L. var. Japonica Hack. Monogr. Androp: 288 ; ct in Bull. Herb. Boiss. sér. 2, III. (1903)501; Rendle 1. c. 361. 蕉州

Rendle distinguishes 3 var. namely : genuina, fasciculata and japonica; and states that var. japonica is scarcely distinguishable from var. fasciculata. However, after Hackel the distinction between the two though slight is as follows:

Spiculis cum callo $2 \mathrm{~mm}$. longo glabro $6-7 \mathrm{~mm}$. longis . . var. fasciculata Hack.

Spiculis cum callo $1 \mathrm{~mm}$. longo glabro rectangulo minus distincto $6-7 \mathrm{~mm}$. longis . . . . . . var. japonica Hack.

Sorghum falvum Beauv.; Rendle 1. c. $367 ;=$ Andropogon serratus, Thunb. var. genuinus Hack. Monogr. Androp. 521 ; et in Bull. Herb. Boiss. VII. (1899)642 et sér. 2, III (1903)501; Hook. f. Fl. Brit. Ind. VII. 185. 蘇州

Spodiopogon cotulifer Hack. Monogr. Androp. 187 et in Bull. Herb. Boiss. VII. (1899) 461, et sér. 2, III. (1908)501; Hook. f. Fl. Brit. Ind. VII. 108; Rendle 1. c. 351. 蘇州

Themeda triandra Forsk. (=Th. Forskali Hack.) var. major subvar. japonica Hack. Monogr. Androp. 662; Komarov in Aet. Hort. Petrop. XX. (1901)251; Rendle 1. c. $378 ;=T h$. Forskali in Bot. Mag. Tokyo XX. 236. 蕉州 
Lygodium japonicum Sw. ; Bot. Mag. Tokyo XX. 177. 蘇州 Nephrodium glanduligerum Makino in Bot. Mag. Tokyo (1896) $58 ;=N$. gracilescens Hook. Synop. Fil 262 ; Diels in Engl. Bot. Jahrb. XXIX. $199 ;=N$. gracilescens var. glanduligerum Bak., Hook. Synop. Fil. 262. 蘇州

Pteridium aquilinum (L.) KUHN; Diels 1 c. 202. 上方山

Pteris serrulata L. fil.; Bot. Mag. Toky). XX. 178 天本山 靈䉷山 